\title{
First-Trimester Uterine Artery Doppler for the Prediction of Preeclampsia in Nulliparous Women: The Great Obstetrical Syndrome Study
}

\author{
Suzanne Demers, MD, MSc ${ }^{1,2}$ Amélie Boutin, $\mathrm{PhD}^{2}$ \\ Mario Girard, $\mathrm{RT}^{2}$ Emmanuel Bujold, MD, $\mathrm{MSC}^{1,2}$ \\ 1 Department of Gynecology, Obstetrics and Reproduction, Faculty of
Medicine, Université Laval, Quebec City, Quebec, Canada
2 Reproduction, Mother and Child Health Unit, CHU de Québec-
Université Laval Research Center, Quebec City, Quebec, Canada
${ }^{3}$ Department of Social and Preventive Medicine, Faculty of Medicine,
Université Laval, Quebec City, Quebec, Canada
}

Cédric Gasse, MD, MSc ${ }^{2,3}$ Olivier Drouin, $\mathrm{MD}^{1}$

\author{
Olivier Drouin, MD
}

Am J Perinatol 2019;36:930-935.

\begin{abstract}
Address for correspondence Emmanuel Bujold, MD, MSc, Department of Obstetrics and Gynecology, Faculty of Medicine, Université Laval, CHU de Québec, 2705, Boulevard Laurier, T3-67, Québec City G1V 4G2, QC, Canada (e-mail: emmanuel.bujold@crchudequebec.ulaval.ca).
\end{abstract}

\begin{abstract}
Keywords

- pregnancy

- preeclampsia

- ultrasound

- Doppler's

- antenatal screening

Objective This study aimed to estimate the performance of first-trimester uterine artery $(\mathrm{UtA})$ pulsatility index $(\mathrm{PI})$ for the prediction of preeclampsia $(\mathrm{PE})$.

Study Design We conducted a prospective cohort study of nulliparous women with singleton gestation at 11 to $13^{6 / 7}$ weeks. UtA-Doppler's was performed on both UtAs and the mean UtA-PI was reported in multiple of median (MoM) adjusted for gestational age. Using receiver operating characteristic curves and their area under the curves (AUC); we calculated the performance of UtA-PI for the prediction of PE. Proportional hazard models were used to develop prediction models combining UtA-PI and maternal characteristics.

Results Out of 4,676 participants with completed follow-up, 232 (4.9\%) developed PE, including $202(4.3 \%)$ term and $30(0.6 \%)$ preterm PE. Mean UtA-PI decreased with gestational age between 11 and $13^{6 / 7}$ weeks $(p<0.001)$. First-trimester UtA-PI was associated with preterm (AUC: $0.69 ; 95 \% \mathrm{Cl}$ [confidence interval]: $0.57-0.80$ ) but not with term (AUC: 0.52 ; $95 \% \mathrm{Cl}$ : 0.48-0.56) PE. UtA-PI combined with maternal characteristics could predict $45 \%$ of preterm PE at a false positive rate of $10 \%$.

Conclusion First-trimester UtA-PI decreases with gestational age between 11 and $13^{6 / 7}$ weeks and is associated with the risk of preterm but not term PE.
\end{abstract}

Preeclampsia (PE) is a multisystem disorder of pregnancy defined by the combination of new-onset hypertension and proteinuria that contribute substantially to perinatal morbidity and mortality worldwide. ${ }^{1,2}$ The etiology of PE remains controversial but it is now recognized that alterations in the growth and development of placental villi and their underlying vasculature play an important role in the pathogenesis of the disease. ${ }^{3}$ The physiological transformation of uterine spiral arteries by the cytotrophoblast invading the myometrium is typically altered in the preterm forms of

received

November 13, 2017

accepted after revision

September 12, 2018

published online

November 10, 2018 pre-eclampsia. ${ }^{4,5}$ Recent evidences suggest that low-dose aspirin started before 16 weeks of gestation can prevent preterm pre-eclampsia. ${ }^{6-10}$ Currently, low-dose aspirin is recommended in high-risk women based on previous pregnancy complications or other risk factors such as chronic hypertension. However, most cases of pre-eclampsia occur in nulliparous women without such risk factors.

Uterine artery (UtA) pulsatility index (PI) measured by Doppler's ultrasound allows an indirect measure of placental vascular resistance which is usually increased in cases of

Copyright @ 2019 by Thieme Medical Publishers, Inc., 333 Seventh Avenue, New York, NY 10001, USA. Tel: +1(212) 584-4662.
DOI https://doi.org/ 10.1055/s-0038-1675209. ISSN 0735-1631. 
incomplete transformation of uterine spiral arteries. Therefore, UtA-PI can be used to estimate the risk of PE. ${ }^{11-14}$ In the second and third-trimester of pregnancy, UtA-PI is strongly related to the risk of PE and fetal growth restriction (FGR). ${ }^{12}$ Some studies evaluated the role of UtA-Doppler's for prediction of $\mathrm{PE}$ in the first-trimester with controversial results. ${ }^{15-21}$ According to Martin et al, UtA-PI greater than the $95 \%$ centile at $11-13$ weeks could predict up to $27 \%$ of PE and $60 \%$ of PE requiring delivery before 32 weeks. ${ }^{15}$ In the meta-analysis of Cnossen et al published in 2008 including 3 studies and 4,966 low-risk women, first-trimester UtA-PI had a sensitivity of $25 \%$ for PE for a specificity of $95 \%{ }^{12}$ In 2009, Poon et al observed that adding maternal characteristics to UtA PI could increase the sensitivity to $45 \%$ for all PE and to $80 \%$ for early-onset PE. ${ }^{22}$ In women with previous PE, first-trimester UtA-PI was shown to discriminate those at high from those at low-risk for recurrence of PE and other adverse pregnancy outcomes, such as FGR and fetal death. ${ }^{23}$ On the other hand, Audibert et al observed no significant association between first-trimester mean UtA-PI and the risk of PE in nulliparous women. ${ }^{20}$

We aimed to evaluate the performance of first-trimester UtA-PI sampled at the level of internal cervical os, as recommended from the International Society of Ultrasound in Obstetrics and Gynecology (ISUOG) for the prediction of PE and preterm PE in nulliparous women.

\section{Materials and Methods}

We conducted a prospective cohort of nulliparous women (great obstetrical syndrome study) at the CHU de QuebecUniversité Laval between March 2011 and December 2014. Women with a singleton pregnancy were recruited between $11^{0 / 7}$ and $13^{6 / 7}$ weeks. Women with crown-rump length (CRL) below $45 \mathrm{~mm}$ or over $85 \mathrm{~mm}$ at the time of ultrasound examination were excluded. We excluded fetal demises up to 20 weeks of gestation, fetal structural anomalies, or chromosomal anomalies leading to medical termination of pregnancy. We also excluded women who reported taking daily aspirin over the course of their pregnancy. Informed consent was obtained from all women prior to study participation. The study was approved by the research ethic board of the CHU de Québec-Université Laval.

At recruitment, a research nurse evaluated eligibility, collected data on maternal characteristics (age, ethnicity, smoking status, history of chronic disease, and use of assisted reproductive technologies), and measured the participant's weight and height for the calculation of body mass index (BMI). Transabdominal ultrasound was conducted by technicians certified from the Fetal Medicine Foundation (FMF) using a Voluson E8 (GE Healthcare). CRL was measured and UtA-Doppler's was performed on both uterine sides to measure and to calculate mean UtA-PI according to the ISUOG criteria. ${ }^{24}$ Mean UtA-PI was log-transformed to obtain normal distribution and reported in multiple of median (MoM) adjusted for gestational age.

Medical charts were reviewed for pregnancy outcomes (PE, FGR, fetal death) after delivery by research nurses blinded to first-trimester data. A maternal-fetal medicine subspecialist blinded to first-trimester data reviewed all cases of gestational hypertension, PE, preterm birth, and low birth weight to confirm all diagnoses.

For descriptive purpose, we reported median and interquartile (IQ) for continuous variables. Wilcoxon's/MannWhitney tests were used to compare characteristics of participants with PE to unaffected pregnancies. Receiver operating characteristic (ROC) curves were constructed based on cumulative incidences computed through proportional hazard models. In these models, we considered all women were at risk of developing PE given a sufficiently long duration of gestation. ${ }^{25}$ Therefore in this conceptual frame, delivery before observation of PE was a censored observation. For the analysis of preterm PE, cases of term PE were hence kept in the population under observation but did not experience the outcome during the "at-risk" period (before 37 weeks of gestation). A linear regression was constructed to look at the relation between CRL and UtAPI. Additionally, we constructed linear models including a regression term for PE or preterm PE and provided figures illustrating the relation. We also explored the association between UtA-PI and gestational age at delivery and PE using generalized additive models with a smoothing spline. We calculated the screening performance (area under the curve [AUC]) of $\log _{10}$ UtA-PI MoMs for the prediction of PE, term $\mathrm{PE}$, and preterm $\mathrm{PE}(<37$ weeks), as well as detection rates (DR) with $10 \%$ false-positive rate (FPR). Additionally, ROC curves combining UtA-PI and maternal characteristics (age, BMI, ethnicity, smoking, history of diabetes, chronic hypertension, chronic renal disease, inflammatory disease, antiphospholipid syndrome, and use of assisted reproductive technologies). Family history of PE was not collected and therefore could not be included. Analyses were conducted with complete observations. We reported effect sizes and their $95 \%$ confidence intervals (CI); a type-I error of 5\% was considered. All analyses were conducted using SAS statistical software packages (Version 9.3, SAS Institute Inc., Cary, NC).

\section{Results}

Out of 4,738 nulliparous women recruited at a mean gestational age of $13.0 \pm 0.6$ weeks ( - Table 1 ), 4,676 (98.7\%) had complete follow-up. We observed 232 (4.9\%) cases of PE, of which $30(0.6 \%)$ occurred before term. Mean UtA-PI values decreased with gestational age $(p<0.001 ;-$ Fig. 1). Women who developed term or preterm PE had a greater BMI than those who did not develop PE (- Table 1).

We observed no difference in terms of raw or MoM UtA-PI values when we compared women who developed term PE compared with the controls, while women who developed preterm PE had higher mean UtA-PI value compared with the controls ( $\boldsymbol{- T a b l e ~} \mathbf{1})$. This trend was similar from 11 to 13 weeks ( - Fig. 2 A and $\mathbf{B}$ ). Looking more specifically to the mean UtA-PI according to gestational age at delivery. We observed that higher first-trimester $\log _{10}$ UtA-PI MoM values were associated with the early forms of PE but not 
Table 1 Characteristics of participants according to the pregnancy outcome

\begin{tabular}{|l|l|l|l|l|l|}
\hline & No PE $(\boldsymbol{n}=4,444)$ & Term PE $(\boldsymbol{n}=\mathbf{2 0 2})$ & $\boldsymbol{p}$-Value ${ }^{\mathrm{a}}$ & Preterm PE $(\boldsymbol{n}=30)^{p}$-Value ${ }^{\mathrm{a}}$ \\
\hline Maternal age, years & $28.7(26.1-31.3)$ & $29.2(26.5-32.0)$ & 0.10 & $29.0(26.0-31.2)$ & 0.97 \\
\hline BMI & $23.7(21.6-26.9)$ & $25.5(22.6-30.3)$ & $<0.0001$ & $26.3(22.9-30.9)$ & 0.01 \\
\hline Gestational age at recruitment, wk & $13.1(12.6-13.4)$ & $13.1(12.6-13.4)$ & 0.95 & $12.9(12.3-13.0)$ & 0.006 \\
\hline Gestational age at delivery, wk & $39.9(39.0-40.7)$ & $39.0(38.1-40.0)$ & $<0.0001$ & $35.1(31.0-36.1)$ & $<0.0001$ \\
\hline Birth weight, grams & $3,317(3,044-3,613)$ & $3,098(2,775-3,500)$ & $<0.0001$ & $2,280(1,420-2,430)$ & $<0.0001$ \\
\hline Mean uterine artery PI (raw values) & $1.6(1.3-2.0)$ & $1.6(1.3-2.0)$ & 0.12 & $2.2(1.8-2.7)$ & $<0.0001$ \\
\hline Log $_{10}$ mean UtA-PI (MoM) & $1.0(0.6-1.4)$ & $1.0(0.4-1.4)$ & 0.11 & $1.4(1.1-1.9)$ & 0.0004 \\
\hline
\end{tabular}

Abbreviations: BMI, body mass index; MoM, multiple of median; PE, preeclampsia; PI, pulsatility index; UtA-PI, uterine artery-PI. Note: Median with their interquartile range.

aWilcoxon's/Mann-Whitney test comparing groups of PE with no PE (controls).

with preterm delivery cases that were not related to PE (-Fig. 3).

The relationship between $\log _{10}$ UtA-PI MoMs and PE-risk varied with time. The association was stronger with preterm cases of PE than term cases. In time-dependent models, the coefficient of the association between UtA-PI and PE for the time period before 37 weeks was 1.09 (standard error [SE] 0.30 ) against -0.22 (SE 0.11), subsequently.

Using ROC curves, we observed that mean UtA-PI MoM was not associated with term PE (AUC $=0.52$; 95\% CI: 0.480.56 ) but was associated with preterm PE (AUC $=0.69 ; 95 \%$ CI: $0.59-0.78)$. At false-positive rates of $10 \%$, UtA-PI alone could have predicted $16 \%$ of term PE and $40 \%$ of preterm PE.
The combination of maternal characteristics and UtA-PI led to significant improvement of all prediction models ( - Fig. 4A, 4B). At false-positive rates of $10 \%$, such models could have predicted $25 \%$ of term and $45 \%$ of preterm PE.

\section{Discussion}

We observed that first-trimester mean UtA-PI is associated with the risk of preterm PE but not with the risk of term PE in nulliparous women. Combined with maternal characteristics, UtA-Doppler's could predict about half of the preterm PE. Our findings suggest that UtA-PI should be included in first-trimester models for the prediction of preterm PE.

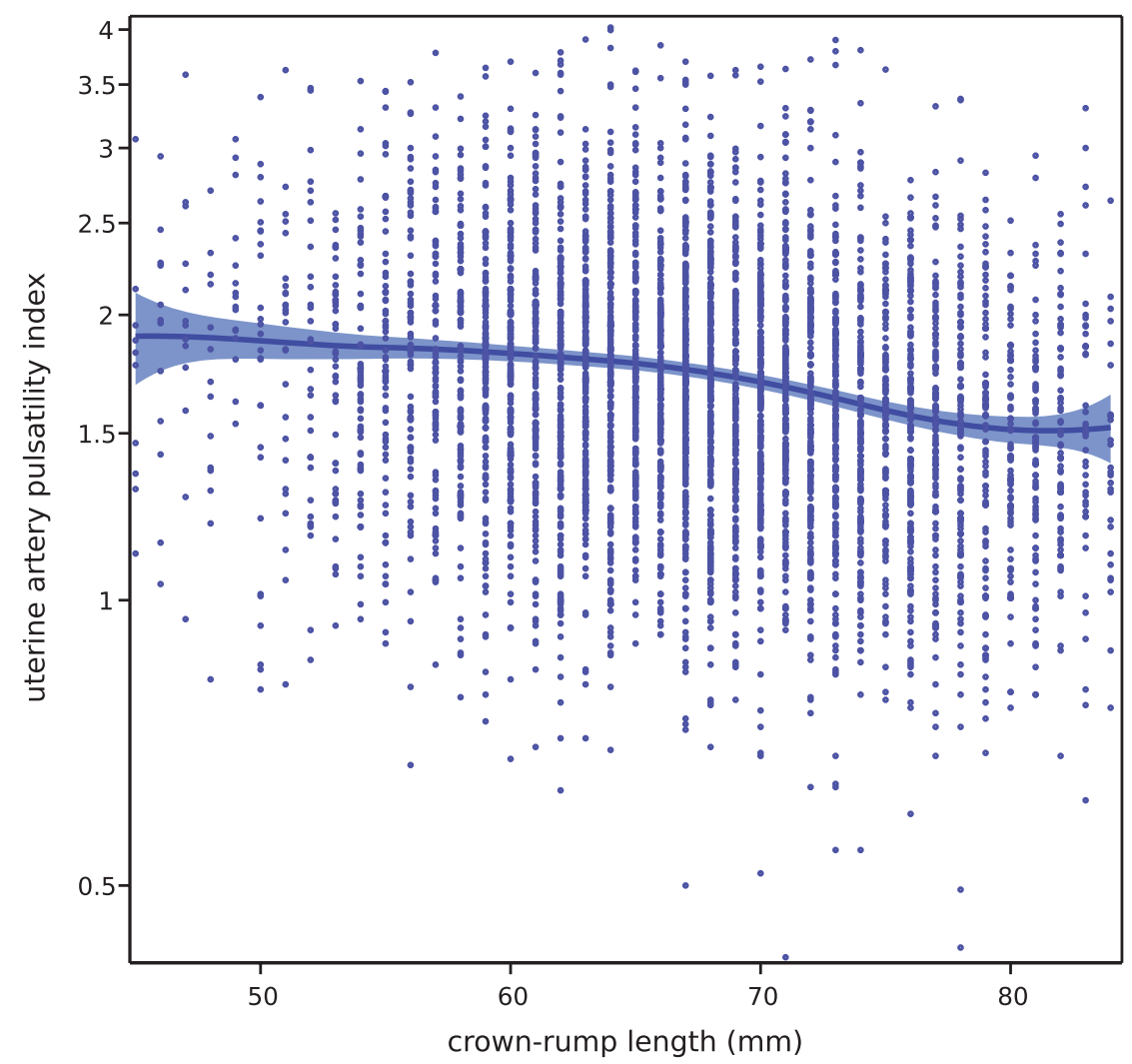

Fig. 1 Uterine artery pulsatility index according to crown-rump length. We observed that the median uterine pulsatility index decreases with crown-rump length $(p<0.001)$. 


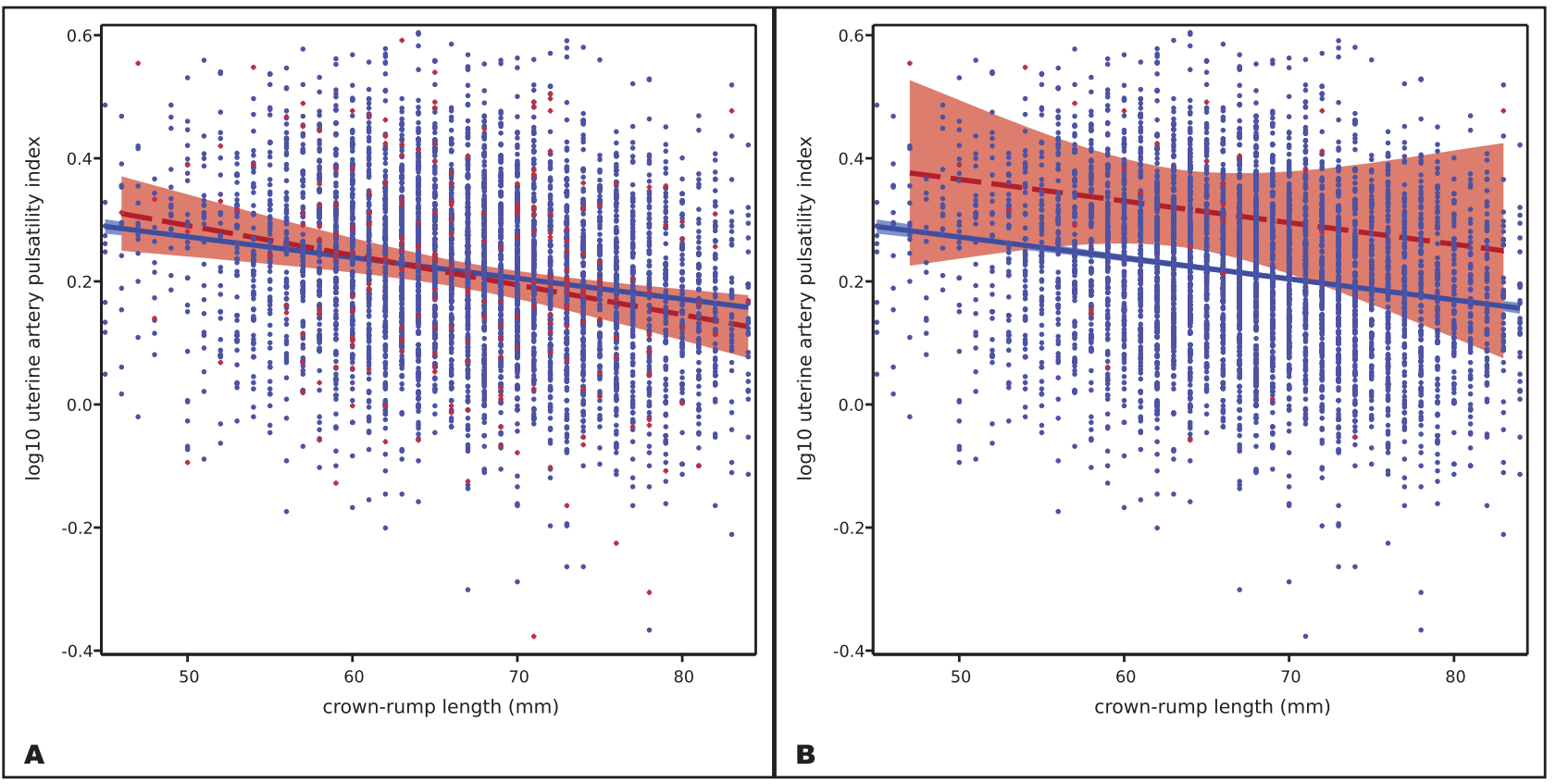

Fig. 2 Uterine artery pulsatility index according to crown-rump length at measurement in women who developed or not preeclampsia. We observed no difference of uterine artery pulsatility index between women who developed (red) and those who did not develop (blue) preeclampsia (A). On the other hand, we observed significant higher values of uterine artery pulsatility index in women who developed preterm preeclampsia (red) compared with those who did not developed preterm preeclampsia (blue, B).

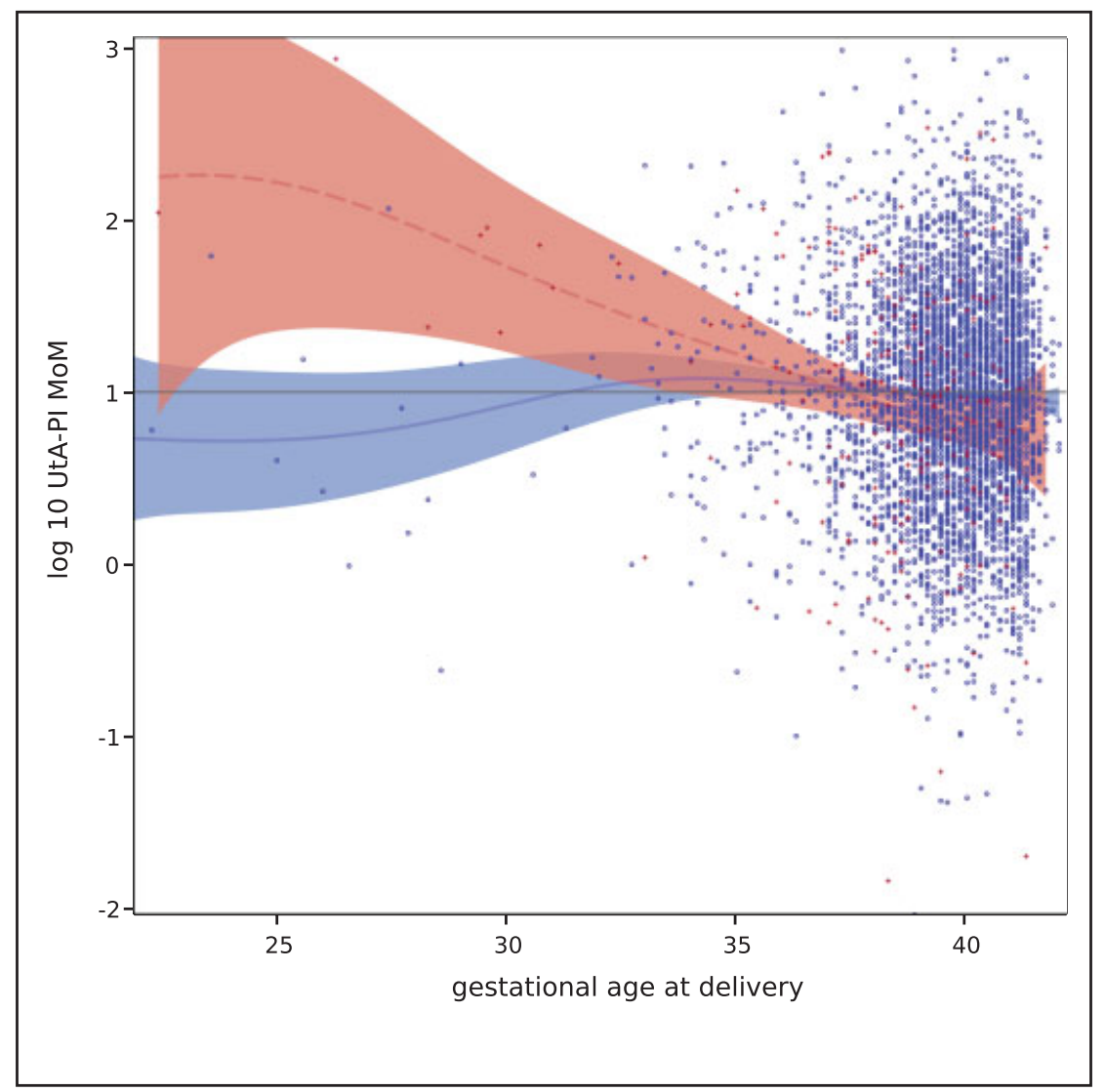

Fig. 3 First-trimester uterine artery pulsatility index in relation with gestational age at delivery in women who developed or not preeclampsia. We observed that first-trimester uterine artery pulsatility index was higher mainly in the early but not in the term forms of preeclampsia and that such increase was not observed in women who delivered before term without preeclampsia. Curves corresponding to penalized B-splines with $95 \%$ confidence bands; preeclampsia cases are in red and the controls in blue. UtA-PI, Uterine artery pulsatility index. 


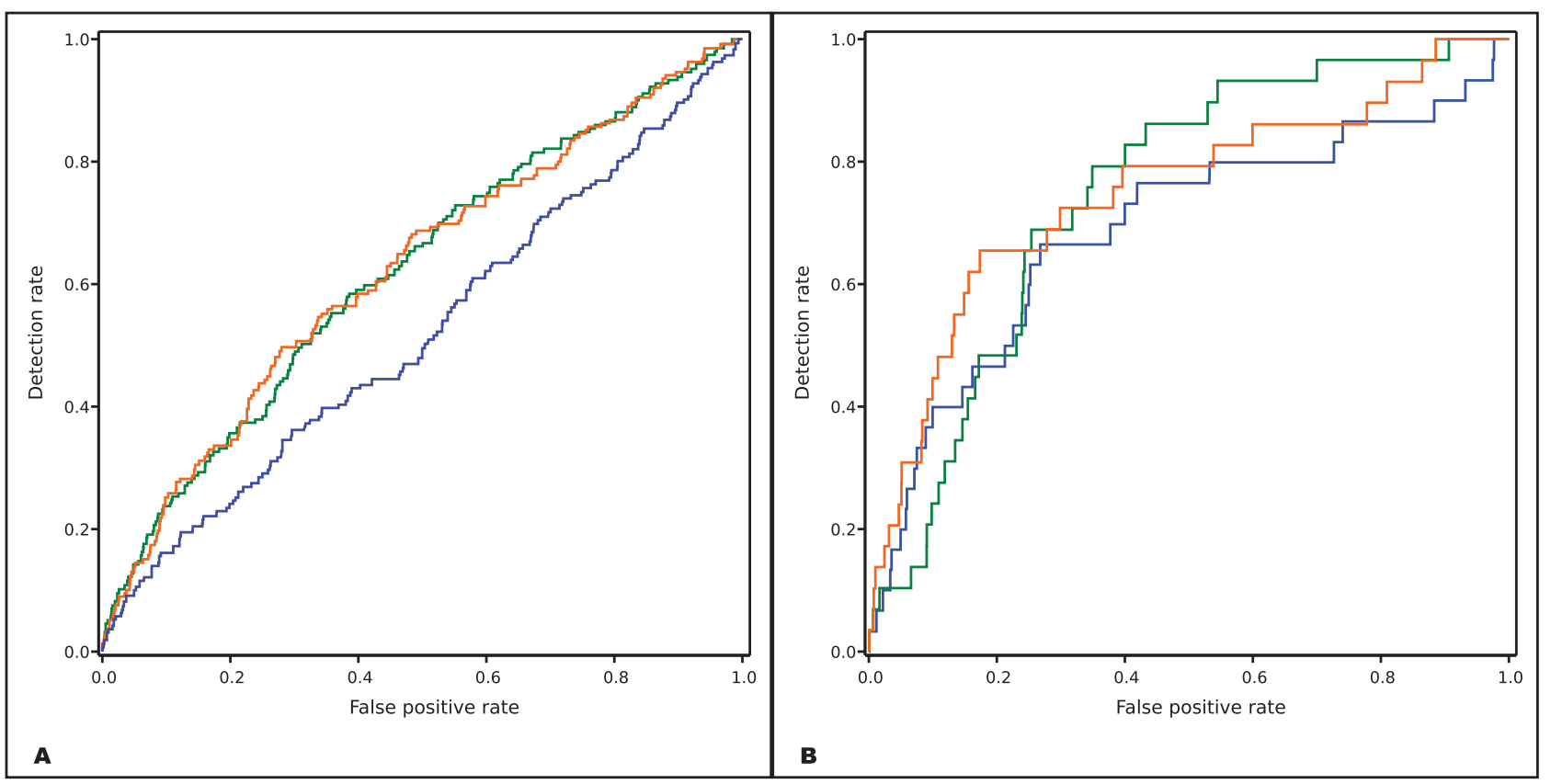

Fig. 4 Prediction of term and preterm preeclampsia with first-trimester uterine artery pulsatility index alone or in combination with maternal characteristics. The figures depict the receiver-operator characteristics curves for the prediction of term (A) and preterm (B) preeclampsia using uterine artery pulsatility index (UtA-PI) alone (blue); maternal characteristics (green); or combination of UtA-PI and maternal characteristics (orange). In figure 4A, we observed a poor prediction of term preeclampsia using UtA-PI alone (AUC: $0.52 ; 95 \% \mathrm{Cl}: 0.48-0.56$ ) or in combination with maternal characteristics (AUC: 0.62; $95 \% \mathrm{Cl}: 0.57-0.67$ ), while in figure $4 \mathrm{~B}$, we observed a significant prediction of preterm preeclampsia using UtA-PI alone (AUC: $0.69 ; 95 \% \mathrm{Cl}: 0.58-0.78)$ that was improved with the addition of maternal characteristics (AUC: $0.75 ; 95 \% \mathrm{Cl}: 0.64-0.86$ ). AUC, area under the curves; $\mathrm{Cl}$, confidence interval.

Our results are in agreement with those of Melchiorre et al who observed that first-trimester UtA-PI is predictive of preterm but not term PE. ${ }^{16}$ They are also in agreement with those of Poon et al who observed a strong association and similar predictive values when using mean UtA-PI in the first-trimester. ${ }^{22}$ The current study and the one of Poon et al observed an improvement of the predictive values with the addition of maternal characteristics into the model. ${ }^{22}$ Further studies confirmed that such prediction of preterm PE can be even further improved by the addition of biochemical markers. ${ }^{26}$ Poon et al did not stratify according to parity but our study confirmed that such prediction model is also useful in nulliparous women. Audibert et al observed that first-trimester maternal characteristics and biochemical markers could be used to predict PE, and mainly the preterm forms of the disease but they did not find any additional benefits to mean UtA-PI. ${ }^{20}$ However, this study was limited by its size ( 893 participants) and by the fact that UtADoppler's was sampled at the crossing of external iliac vessels in opposition with Doppler's acquired at the level of internal cervical os in the first-trimester, as it is now recommended by the FMF and the ISUOG. ${ }^{24}$ Lefebvre et al observed that first-trimester UtA-PI collected at the level of the internal cervical os correlated better with mid-trimester UtA-PI than UtA-PI collected at the level of the apparent crossover with the external ilia vessels. ${ }^{27}$ Finally, our results are in agreement with the meta-analysis of Velauthar et al suggesting that first-trimester UtA-PI is highly associated with the early-onset form of PE making it a useful tool in the early detection of women who could benefit from aspirin prophylaxis. ${ }^{28}$ From literature and the current study, we could conclude that first-trimester UtA-PI is likely to be useful in the prediction of preterm PE in nulliparous women, when collected at the level of internal cervical os, when adjusted for gestational age, and when combined with maternal characteristics and ideally with additional biochemical markers such as pregnancy-associated plasma protein A (PAPP-A) and placental growth factor (PIGF).

The current study has some limitations. First, UtA-Doppler's were performed by a limited numbers of certified ultrasound technicians with strong experience in UtA-PI. It is possible that similar predictive values required a minimum of training and experience from the sonographers. Ridding et al demonstrated that simple audits could significantly improve the quality and reproducibility of the measurements. ${ }^{29}$ The same group also found that a small discrepancy in the sampling level (as less than $2 \mathrm{~cm}$ ) could lead to significant change in UtA-PI values, stressing the importance of following the techniques criteria. ${ }^{29}$ Second, we did not collect information regarding family history of PE. It is possible that such information could improve the predictive value of a model combining UtA-PI and maternal characteristics.

With the recent publication of the ASPRE trial confirming the important reduction of preterm PE with low-dose aspirin when started at 11 to 13 weeks in high-risk women, it is very important to determine the optimal screening test in each population. ${ }^{10}$ The current study confirms that mean UtA-PI should not be used alone but remains a useful factor in the first-trimester prediction of preterm PE. It should be adjusted for gestational age (or CRL) at the time of measurement and combined with other markers (maternal characteristics and biochemical markers) to reach optimal benefits. 
Funding

This project was funded by the Jeanne-et-Jean-Louis-Lévesque perinatal research Chair at Université Laval and by the Jean-Louis-Lévesque Foundation, Montreal, Quebec, Canada. Dr Emmanuel Bujold holds a Clinician-Scientist Award from the Fonds de recherche du Québec-Santé (FRQ-S).

\section{Conflict of Interest}

None.

\section{Acknowledgments}

We thank the members of the Department of Obstetrics \& Gynecology and the Department of Family Medicine of the CHU de Québec-Université Laval, Quebec, Canada for their collaboration in the recruitment of women in this study.

\section{References}

1 World Health Organization International Collaborative Study of Hypertensive Disorders of Pregnancy. Geographic variation in the incidence of hypertension in pregnancy. Am J Obstet Gynecol 1988;158(01):80-83

2 Duley L. The global impact of pre-eclampsia and eclampsia. Semin Perinatol 2009;33(03):130-137

3 Steegers EA, von Dadelszen P, Duvekot JJ, Pijnenborg R. Pre-eclampsia. Lancet 2010;376(9741):631-644

4 Brosens I, Pijnenborg R, Vercruysse L, Romero R. The "Great Obstetrical Syndromes" are associated with disorders of deep placentation. Am J Obstet Gynecol 2011;204(03):193-201

5 Ogge G, Chaiworapongsa T, Romero R, et al. Placental lesions associated with maternal underperfusion are more frequent in early-onset than in late-onset preeclampsia. J Perinat Med 2011; 39(06):641-652

6 Bujold E, Roberge S, Lacasse Y, et al. Prevention of preeclampsia and intrauterine growth restriction with aspirin started in early pregnancy: a meta-analysis. Obstet Gynecol 2010;116(2, Pt 1):402-414

7 Roberge S, Giguère Y, Villa P, et al. Early administration of lowdose aspirin for the prevention of severe and mild preeclampsia: a systematic review and meta-analysis. Am J Perinatol 2012;29 (07):551-556

8 Roberge S, Villa P, Nicolaides K, et al. Early administration of lowdose aspirin for the prevention of preterm and term preeclampsia: a systematic review and meta-analysis. Fetal Diagn Ther 2012;31(03):141-146

9 Roberge S, Nicolaides K, Demers S, Hyett J, Chaillet N, Bujold E. The role of aspirin dose on the prevention of preeclampsia and fetal growth restriction: systematic review and meta-analysis. Am J Obstet Gynecol 2017;216(02):110-120

10 Rolnik DL, Wright D, Poon LC, et al. Aspirin versus placebo in pregnancies at high risk for preterm preeclampsia. N Engl J Med 2017;377(07):613-622

11 Espinoza J, Romero R, Mee Kim Y, et al. Normal and abnormal transformation of the spiral arteries during pregnancy. J Perinat Med 2006;34(06):447-458

12 Cnossen JS, Morris RK, ter Riet G, et al. Use of uterine artery Doppler ultrasonography to predict pre-eclampsia and intrauterine growth restriction: a systematic review and bivariable meta-analysis. CMAJ 2008;178(06):701-711
13 Papageorghiou AT, Yu CK, Nicolaides KH. The role of uterine artery Doppler in predicting adverse pregnancy outcome. Best Pract Res Clin Obstet Gynaecol 2004;18(03):383-396

14 Poon LC, Volpe N, Muto B, Yu CK, Syngelaki A, Nicolaides KH. Second-trimester uterine artery Doppler in the prediction of stillbirths. Fetal Diagn Ther 2013;33(01):28-35

15 Martin AM, Bindra R, Curcio P, Cicero S, Nicolaides KH. Screening for pre-eclampsia and fetal growth restriction by uterine artery Doppler at 11-14 weeks of gestation. Ultrasound Obstet Gynecol 2001;18(06):583-586

16 Melchiorre K, Wormald B, Leslie K, Bhide A, Thilaganathan B. Firsttrimester uterine artery Doppler indices in term and preterm preeclampsia. Ultrasound Obstet Gynecol 2008;32(02):133-137

17 Plasencia W, Maiz N, Poon L, Yu C, Nicolaides KH. Uterine artery Doppler at $11+0$ to $13+6$ weeks and $21+0$ to $24+6$ weeks in the prediction of pre-eclampsia. Ultrasound Obstet Gynecol 2008; 32(02):138-146

18 Rizzo G, Capponi A, Cavicchioni O, Vendola M, Arduini D. First trimester uterine Doppler and three-dimensional ultrasound placental volume calculation in predicting pre-eclampsia. Eur J Obstet Gynecol Reprod Biol 2008;138(02):147-151

19 Poon LC, Staboulidou I, Maiz N, Plasencia W, Nicolaides KH. Hypertensive disorders in pregnancy: screening by uterine artery Doppler at 11-13 weeks. Ultrasound Obstet Gynecol 2009;34(02):142-148

20 Audibert F, Boucoiran I, An N, et al. Screening for preeclampsia using first-trimester serum markers and uterine artery Doppler in nulliparous women. Am J Obstet Gynecol 2010;203(04):383. e1-383.e8

21 Kuc S, Wortelboer EJ, van Rijn BB, Franx A, Visser GH, Schielen PC. Evaluation of 7 serum biomarkers and uterine artery Doppler ultrasound for first-trimester prediction of preeclampsia: a systematic review. Obstet Gynecol Surv 2011;66(04):225-239

22 Poon LC, Karagiannis G, Leal A, Romero XC, Nicolaides KH. Hypertensive disorders in pregnancy: screening by uterine artery Doppler imaging and blood pressure at 11-13 weeks. Ultrasound Obstet Gynecol 2009;34(05):497-502

23 Demers S, Bujold E, Arenas E, Castro A, Nicolaides KH. Prediction of recurrent preeclampsia using first-trimester uterine artery Doppler. Am J Perinatol 2014;31(02):99-104

24 Salomon LJ, Alfirevic Z, Bilardo CM, et al. ISUOG practice guidelines: performance of first-trimester fetal ultrasound scan. Ultrasound Obstet Gynecol 2013;41(01):102-113

25 Wright D, Akolekar R, Syngelaki A, Poon LC, Nicolaides KH. A competing risks model in early screening for preeclampsia. Fetal Diagn Ther 2012;32(03):171-178

26 O'Gorman N, Wright D, Syngelaki A, et al. Competing risks model in screening for preeclampsia by maternal factors and biomarkers at 11-13 weeks gestation. Am J Obstet Gynecol 2016;214(01):103. e1-103.e12

27 Lefebvre J, Demers S, Bujold E, et al. Comparison of two different sites of measurement for transabdominal uterine artery Doppler velocimetry at 11-13 weeks. Ultrasound Obstet Gynecol 2012;40 (03):288-292

28 Velauthar L, Plana MN, Kalidindi M, et al. First-trimester uterine artery Doppler and adverse pregnancy outcome: a meta-analysis involving 55,974 women. Ultrasound Obstet Gynecol 2014;43 (05):500-507

29 Ridding G, Hyett JA, Sahota D, McLennan AC. Assessing quality standards in measurement of uterine artery pulsatility index at 11 to $13+6$ weeks' gestation. Ultrasound Obstet Gynecol 2015;46 (03):299-305 